\title{
Development of fusiform aneurysms induced by topical application of elastase in a rabbit model
}

\author{
Guoquan Jiang ${ }^{1}$, Zifu Li ${ }^{2}$, Xiaochun Jiang ${ }^{3}$, Zhenbao $\mathrm{Li}^{3}$, Shanshui $\mathrm{Xu}^{3}$ and Xinggen Fang ${ }^{3^{*}}$
}

\begin{abstract}
Background: Research on the etiology and pathophysiology of fusiform aneurysm has been impeded due to the inability to collect fusiform aneurysm specimens. We aim to resolve this through the development of a novel fusiform aneurysm model in rabbits.

Methods: Sixty New Zealand White rabbits were divided into ten groups ( $n=6$ per group): groups A, B, C, D, E and groups a, b, c, d, e. Elastase, at a concentration of $0,0.5,1,2.5$ and $5 \mathrm{U} / \mu \mathrm{L}$ respectively was administered to each rabbit to incubate their carotid arteries. Three weeks later, angiography, histomorphometry, immunohistochemistry and immunofluorescent were performed.

Results: Heparin administration is indispensable. No thrombosis was observed in groups A, B, C, D and E, whereas, increased thromboembolism occurred in groups $a, b, c, d$ and e. Based on the size and wall thickness of aneurysms specimens, $5 \mathrm{U} / \mu \mathrm{L}$ was the optimal concentration of elastase to induce fusiform aneurysms. At $5 \mathrm{U} / \mu \mathrm{L}$, the intraluminal carotid diameter increased significantly from $2.50 \pm 0.32 \mathrm{~mm}$ to $3.11 \pm 0.55 \mathrm{~mm}(p<0.01)$. The wall thickness significantly reduced from $176.0 \pm 39.8 \mu \mathrm{m}$ to $39.7 \pm 14.6 \mu \mathrm{m}(p<0.01)$ post aneurysm induction. The histolopathological evaluation revealed the elastic lamina and the smooth muscle cell's lamina were markedly attenuated and the intimal endothelial lamina became thin or even absent.
\end{abstract}

Conclusions: Our research demonstrates that intracranial fusiform aneurysm could be modeled in rabbit carotid artery adventitia incubation by porcine pancreatic elastase.

Keywords: Fusiform aneurysm, Rabbit model

\section{Background}

Intracranial fusiform aneurysms are less frequent in comparison with saccular aneurysms, defined as nonsaccular dilatations that include the entire arterial wall for a short distance rather than pouch from the side of the artery [1]. In the past, plenty of hypotheses about the pathogenesis of intracranial fusiform aneurysms have been proposed, such as atherosclerosis, abnormal collagen and elastin metabolism, dissections, infections and the invasion of the arterial wall by neoplasm [2, 3]. Unfortunately, none of them could explain the pathogenesis absolutely. Besides, the technology and tools available

\footnotetext{
* Correspondence: chali88@163.com

${ }^{3}$ Department of Neurosurgery, First Affiliated Hospital of Wannan Medical College, Wuhu 241000, China

Full list of author information is available at the end of the article
}

for therapeutic treatment need to further improvement. The research on intracranial fusiform aneurysms is inhibited due to the lack of clinical specimen availability in comparison to saccular aneurysms that are often clipped [4]. All these need to develop the model of fusiform aneurysm. To our knowledge, few models have been proposed [5-8]. Moreover, there is a series of unanswered questions in these models i.e. complicated surgical procedures, the small size of aneurysm and the unknown biological effect yielded by the suture lines [8-11].

Elastase has been frequently applied in animals' models of aneurysm induction. The feasibility, safety, and efficacy of these models have been accepted [12-17]. The elastase model has mostly been utilized in the model for induction of saccular aneurysms and only a few groups have attempted development of fusiform aneurysm model. 
Besides, in the few models proposed, complex surgical procedures are required or a high thrombosis rate is observed post aneurysm induction $[6,18]$. Hence, the development of a novel intracranial fusiform aneurysms' model is necessary.

\section{Methods}

All animal experiments were performed in accordance with a protocol approved by the Institutional Animal Care and Use Committee of Wannan Medical College.

\section{Description of the animal model}

New Zealand White rabbits of $2.5-3.0 \mathrm{~kg}(n=60)$ were randomly divided into two large groups $(n=30$ per group). Every large group was divided into five sub groups ( $n=6$ per group). The specimens from the first large group divided into subgroups A, B, C, $\mathrm{D}$ and $\mathrm{E}$ were heparinized. The specimens from the second large group were subdivided into groups a, b, c, d and e without heparin administration. Specimens from groups A, B, C, D and E as well as groups a, b, c, $d$ and e were respectively incubated with porcine pancreatic elastase solution at the concentration of 0 , $0.5,1,2.5$ and $5 \mathrm{U} / \mu \mathrm{L}$.

All rabbits were anesthetized through intravenous injection of $30 \mathrm{mg} / \mathrm{kg}$ sodium pentobarbital. The surgical exposures and creations of the fusiform aneurysms were performed strictly under sterile conditions.

- Step 1: A segment of the right common carotid artery (RCCA), approximately $2.5 \mathrm{~cm}$ in length, was carefully exposed and isolated as previously described [19]. A piece of sterile neurological sponges
$(7 \times 8 \mathrm{~mm})$ and a slice of latex cuff $(1.2 \times 2 \mathrm{~cm})$ were used to isolate the exposed carotid artery (Fig. 1a, d).

- Step 2: The proximal and distal portions of the latex cuff were temporarily ligatured around the carotid artery with 4.0 silk sutures. After that, different concentrations $(0.5,1,2.5$ or $5 \mathrm{U} / \mu \mathrm{L})$ of porcine pancreatic elastase ( $\geq 30$ units/mg, $\mathrm{pH}$ 8.1-8.9, PI 9.5, Shanghai Kayon Biological Technology Co., Ltd., Shanghai, China) solution were perfused into the latex cuff through $24 \mathrm{G}$ venous catheter until the latex cuff was filled entirely to coat the vessel and maintained for $20 \mathrm{~min}$ (Fig. 1b, e).

- Step 3: The elastase solution in the latex cuff was absorbed with sterile cotton gauze 20 min later. The temporary ligatures, neurological sponges and the latex cuff were removed. Then, the arterial segment and incision was irrigated with physiological saline to clear any residual elastase. Finally, the fusiform vessel segment was examined and the surgical site and incision was closed (Fig. 1c, f).

- Specimens from the control groups also underwent the same above mentioned steps. The unique difference is that the carotid artery segments were incubted with physiological saline rather than elastase solution.

All animals in the first large group were administered with heparin $(2000 \mathrm{U} / \mathrm{Kg})$ intravenously after the RCCA were exposed and prior to the installation of the elastase. The same dose was applied once per day for the next 3 days. All animals in the second large group were not administered with heparin. All animal specimens in the study were given antibiotic injections to prevent infections post surgical exposure.

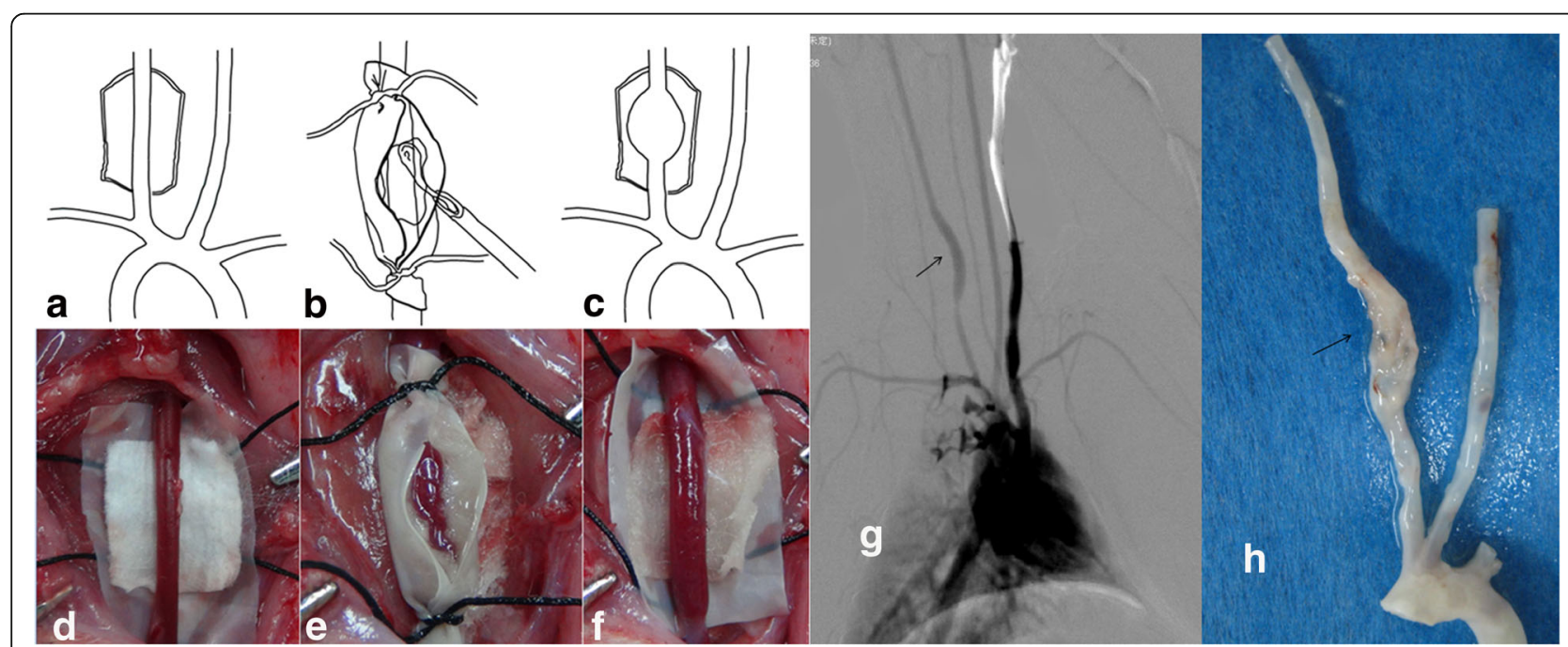

Fig. 1 a-f Schematic presentation of the main steps of the procedure. a, d Step 1 b, e: Step 2 c, $\mathbf{f}$ Step 3. $\mathbf{g}$ IV DSA of a rabbit carotid artery at 21 days after $5 \mathrm{U}$ of porcine pancreatic elastase incubation in the right carotid artery exhibited luminal enlargement (arrow). $\mathbf{h}$ gross specimen of a harvested rabbit's fusiform aneurysm, just as the arrow indicated (original magnification, $\times 1$ ) 


\section{Intravenous digital subtraction angiography}

All rabbits except for the samples lost to attrition underwent an intravenous digital subtraction angiography (IVDSA) through auricular veins 3 weeks post-surgery using 3-5 ml angiographic contrast injections through a 24 gauge venous catheter under anesthesia. The size of aneurysm in our experiment was defined through the inner diameter of the dilated segment (that is the greatest dimension of the transverse minor axis of the fusiform segment). The images and measurements were evaluated by an independent observer who was blinded to other groups.

\section{Histological analysis}

Immediately after IVDSA, all specims were sacrificed through an overdose of sodium pentobarbital $(120 / \mathrm{mg} / \mathrm{kg})$. The specimens from these animals were then acquired after in vivo fixation with $10 \%$ phosphate-buffered formaldehyde solution perfused at $100 \mathrm{mmHg}$ maintained for 20 min. From the proximal to the distal end, each specimen was cut into continuous slices of $5 \mu \mathrm{m}$. Next, these slices were stained with hematoxylin and eosin (H\&E) for general appearance and elastic van-Gieson (EVG) dye for elastin. Images of the sections were analyzed by using Image-pro plus 6.0software. The wall thickness was measured as the average thickness of 10 points along the cross-section area in the H\&E stained sections.

\section{Immunohistochemistry analysis}

Tissue sections in group $\mathrm{E}$ were de-paraffinized in xylene, rehydrated through graded alcohol washes, and incubated with $1 \% \mathrm{H}_{2} \mathrm{O}_{2}$ in methanol maintained for 10 min to block endogenous peroxidase activity. Non-specific binding was blocked with bovine serum (A8020, Solarbio, Beijing, China) for $20 \mathrm{~min}$ at room temperature, and then CD31 (ready-to-use, ab28364, Abcam) antibodies were incubated overnight in a humid chamber at $4{ }^{\circ} \mathrm{C}$ overnight. After a wash in phosphor buffered saline (PBS), sections were incubated with biotinylated anti-mouse second antibody (Boster, Wuhan, China) for $20 \mathrm{~min}$ followed by the SP method according to the manufacture's protocol. Diaminobenzidine tetrahydrochloride was used to visualize the sections and counterstaining with hematoxylin was performed, after that the sample was coverslipped.

\section{Immunofluorescent analysis of smooth muscle cells}

After blocking endogenous peroxidases, sections in group E were incubated at $4{ }^{\circ} \mathrm{C}$ overnight with mouse monoclonal anti-alpha smooth muscle actin (1:150 diluted in PBS,GB11001), followed by incubation with Cy3-conjugated goat anti-mouse IgG (Google Biological Technology Co., Ltd., Wuhan, China) to identify smooth muscle cells (SMCs).

\section{Statistical analysis}

All data were expressed as mean \pm standard deviation. Significant differences between different groups were determined by 2-way ANOVA-test (GraphPad Prism 5.01). A $p$-value of $<0.05$ was considered significant.

\section{Results}

\section{Assessment of heparinization}

In the second large group, seven rabbits were lost to attrition. From the seven expired rabbits, five belonged to group e and two belonged to group d. Post mortem autopsy found that arterial thromboembolism occurred in bilateral common carotid arteries in these seven rabbits. Incidence of right common carotid artery occlusion by thrombosis was reported in six survival rabbits which. In summary, 13 out of $30(43.3 \%)$ rabbits in the nonheparinized groups had parent artery thromboembolism as a result of elastase incubation. The incidence of carotid patency of group a, b, c, d and e were respectively 100, 83.3, 66.7, 33.3 and 0\% (Fig. 2). However, all rabbits samples in the first large group (heparinized) survived without any reports of thrombosis.

\section{Influence of elastase solution on arterial enlargement}

The inner diameters of five groups in the first large group were measured by DSA. As the concentration of elastase increased, the fusiform enlargement of the artery is evident (Fig. 3a): For group A specimens, the inner diameter did not enlarge $(2.50 \pm 0.32 \mathrm{~mm})$. The inner diameter was $2.56 \pm 0.44 \mathrm{~mm}$ for group B specimens which did not enlarge significantly enlarge compared to group A $(P>0.05)$. For group $C$ specimens, the inner diameter was $2.77 \pm 0.49 \mathrm{~mm}$ which was significantly enlarged compared to groups $\mathrm{A}$ and $\mathrm{B}(P<0.05)$. The inner diameter was $2.99 \pm 0.62 \mathrm{~mm}$ for group $\mathrm{D}$ specimens which was significantly enlarged in comparison to groups A, B and $C(P<0.05)$. Finally in group $\mathrm{E}$

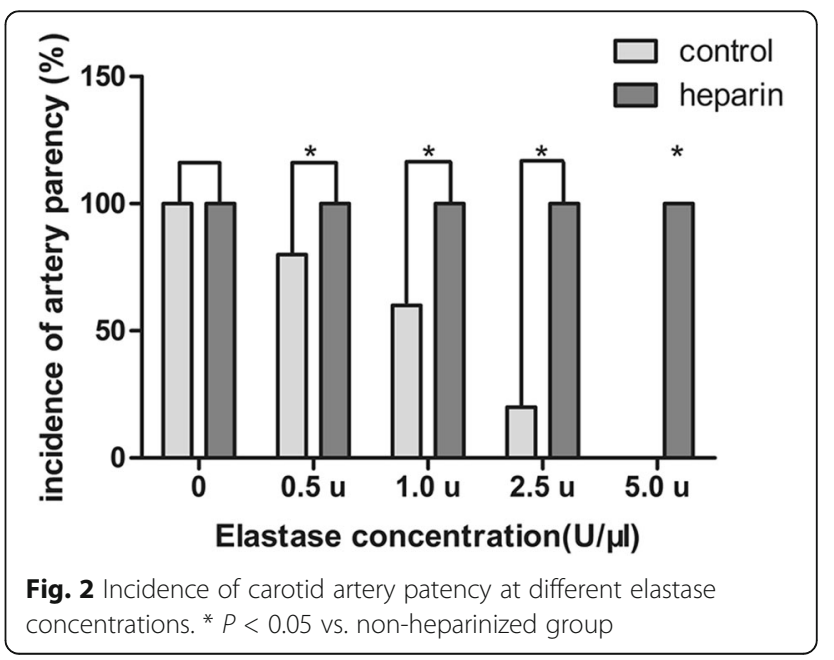




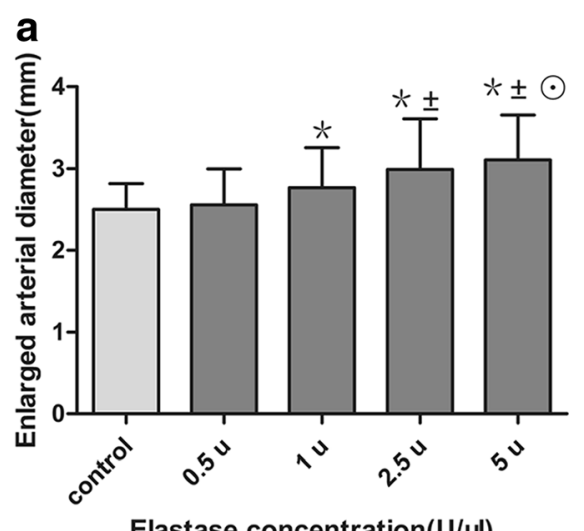

Elastase concentration $(\mathrm{U} / \mu \mathrm{l})$ b

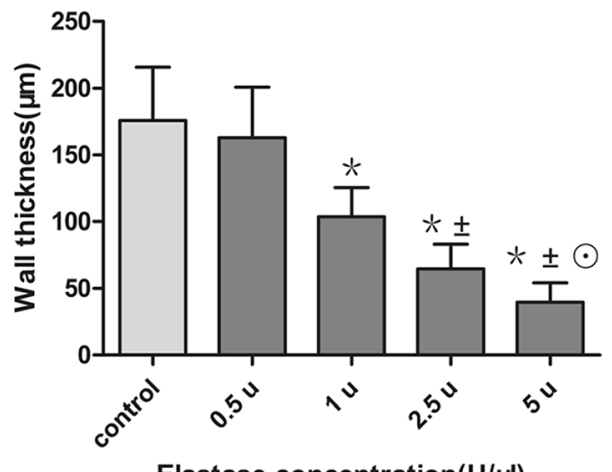

Elastase concentration $(\mathrm{U} / \mu \mathrm{l})$

Fig. 3 a Size of aneurysm (enlarged arterial diameter) in elastase-treated carotid arteries at 4 concentrations (0.5, 1, 2.5 and $5 \mathrm{U}$ ) compared with the control (group A). b Graph representation of the wall thickness in elastase-treated carotid arteries at 4 concentrations of the elastase (0.5, 1, 2.5 and $5 \mathrm{U}$ ) and compared to the control (group A). Results are expressed as means $\pm \mathrm{SD}$. ${ }^{*} P<0.05$ vs. control, $\pm P<0.05$ vs. $0.5 \mathrm{U} ; \odot p<0.05$ vs. $1 \mathrm{U}$

specimens, the inner diameter was $3.11 \pm 0.55 \mathrm{~mm}$, which was significantly enlarged compared to all other groups $(P<0.05)$. These data demonstrated that increase in elastase concentration was linked to incremental enlargement of fusiform artery. So $5 \mathrm{U} / \mu \mathrm{L}$ was optimal concentration which would be applied in the next study on morphology and histology of fusiform aneurysms. The $5 \mathrm{U} / \mu \mathrm{L}$ was the highest concentration of elastase in our experiment since it is challenging to create an elastase solution with higher concentration of elastase.

\section{Change of wall thickness on enlarged arterial segment} Histological examination of groups B -E revealed that the carotid wall thickness on enlarged arterial segment was thinner than group A it is the control group (Fig. 3b): On group A the wall thickness was $176.0 \pm 39.8 \mu \mathrm{m}$ while the wall thickness on groups B-E were respectively $163.1 \pm 37.6 \mu \mathrm{m}, 103.7 \pm 21.8 \mu \mathrm{m}, 64.6 \pm 18.5 \mu \mathrm{m}$ and $39.7 \pm 14.6 \mu \mathrm{m}$. While Group B was not significantly thinner than group A $(P>0.05)$, group $C$ was significantly thinner than group A and group B $(P<0.05)$. Group D decreased significantly compared to groups $\mathrm{A}, \mathrm{B}$ and $\mathrm{C}$ $(P<0.05)$ and group $E$ decreased meaningfully compared to all the other groups $(P<0.05)$.

\section{Specimen and histology}

The specimen was fusiform enlarged and located on the mid-portion of the RCCA when the concentration of elastase was $5 \mathrm{U} / \mu \mathrm{L}$ (Fig. $1 \mathrm{~g}, \mathrm{~h}$ ). H\&E staining demonstrated: the wall of vessel was very thin and the inner lumen enlargement was obvious (Fig. 4b) and the structure of media layer, intima layer and intima-media layer were disorganized and the cellular elements were markedly lost (Fig. 4d). Whereas, on the control groups, the vessel wall was normal (Fig. 4a) with the elastin intact and the structure of medial, intimal and intima-media layers was organized (Fig. 4c). Elastic van Gieson (EVG) staining demonstrated that the elastic network of the internal and external elastic lamina and media layer was severely destroyed in comparison with the intact elastin layers in the normal vessel (Fig. 4e).

\section{Immunohistochemical and immunofluorescence analysis}

The sections immunostained for CD31 showed destruction of the endothelium on the model which the concentration of elastase was $5 \mathrm{U} / \mu \mathrm{L}$ (Fig. 4f). Immunofluorescence staining demonstrated that the thickness of the smooth muscle ( $\alpha$-SMA) cell layer was significantly decreased and the structure of the smooth muscle was disordered (Fig. 4h), in comparison to the normal layer of smooth muscle (Fig. 4g).

\section{Discussion}

In this report, we characterized the morphology and histology of fusiform aneurysms induced in New Zealand white rabbits. The morphological enlargement of the inner lumen of the vessel as well as attenuation of the wall can be distinctly demonstrated. The parent arteries in heparinized groups remained unobstructed without spontaneous thrombosis up to the 21 days follow up. Histologically, the elastic lamina and SMCs were markedly attenuated, the intimal endothelial lamina became apparently thin or even absent. Using these findings, this unique creation of fusiform aneurysms is similar to human aneurysm.

In our opinion, the following factors play key roles in improving the outcome of this experimental aneurysm creation: 1) Careful exposure and preparation of a long segment of the RCCA, without damaging the superior laryngeal nerves and small vessels. 2) The optimal 


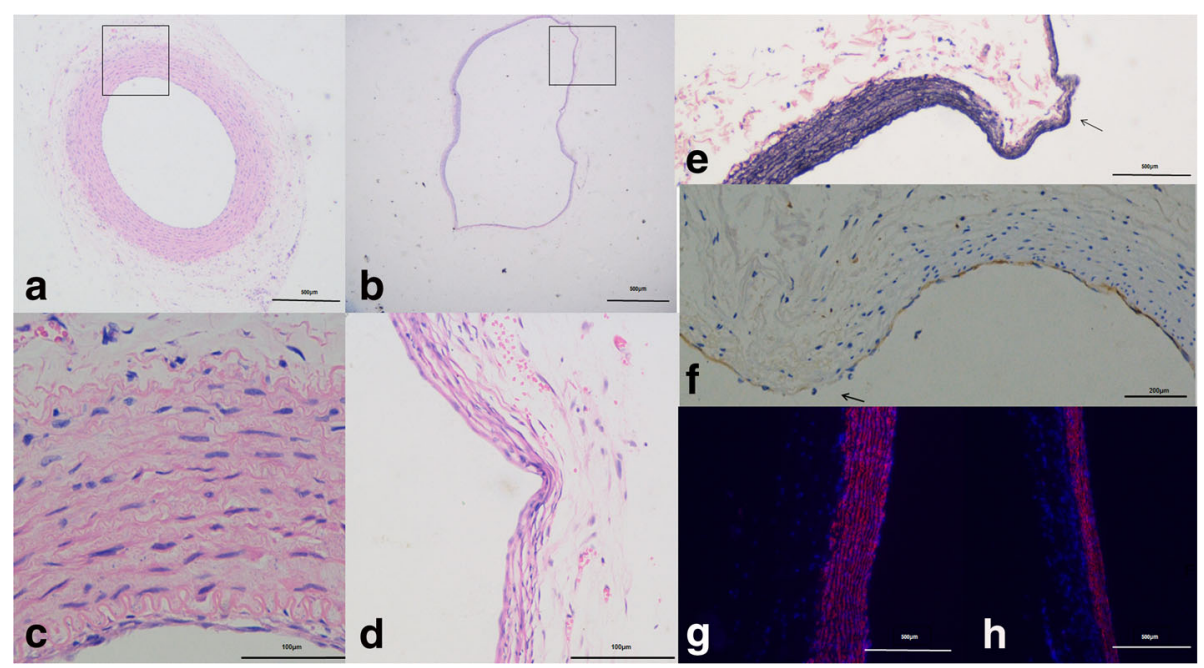

Fig. 4 a-d Histological Analysis of Aneurysm (Transverse sections) H\&E staining shows a thin aneurysm wall and enlarged inner diameter of aneurysm ( $\mathbf{b} \times 40, \mathbf{d} \times 200)$ compared to the normal vessel $(\mathbf{a} \times 40, \mathbf{c} \times 200)$. e-h Elastic Van Gieson staining of Vertical sections (e $\times 40)$, reveals severe destruction of elastic lamina in the aneurysms Elastic fibers (arrow): dark blue to black; nuclei: black; collagen: red; other structures: yellow. Immunohistochemical staining $(\mathbf{f} \times 100)$ shows a lack of intimal endothelial (CD31 (yellow) positive) cells (arrow) in the intimal endothelial (CD31 (yellow) positive) cells (arrow). Immunofluorescence staining demonstrates that the thickening of the smooth muscle (a-SMA (red) positive) cell layer was decreased and the structure of smooth muscle was disordered $(\mathbf{g} \times 40)$, compared to the normal layer of smooth muscle ( $\mathbf{h} \times 40$ )

method of elastase incubation is adventitia incubation rather than intraluminal incubation. The surgical procedure was simple and easy with a low rate of thrombosis. In our experiment, there was no thrombosis in heparinized groups while the thrombosis rate was relatively high (25\%) through intraluminal incubated method according to the report of Reinald et al. [18]. 3) Heparin administration is also indispensable. There was no thrombosis in heparinized groups. While in nonheparinized groups, there was a high rate of thrombosis of parent artery which contributed to the death of the animals. 4) The optimal concentration of elastase is $5 \mathrm{U} / \mu \mathrm{L}$ in our experiment.

There are some advantages in our model: 1) our model has a good stability and durability since these aneurysms are stable for 21 days. 2) The latex cuff application has two distinct advantages: it protects the vessels once they were temporarily ligated and it limits the exposure of elastase to surrounding tissues. 3) The size of these aneurysms is enough in response to therapeutic intervention. To our knowledge, there are some previously described models about fusiform aneurysm [15, 20, 21]. However, these aneurysms are microscopic in scale and not large enough to test new endovascular devices. 4) There is no thrombosis in our models. 5) Our surgical procedures are very simple and facile.

Of course, our model has some limitations as well. Evidently the induced aneurysms are located on extracranial vessel and not on intracranial vessel.

\section{Conclusions}

Our model is significant because the acquired fusiform aneurysms are not only in line with the humans' on morphology but also on histology. We have replicated the intracranial fusiform aneurysm through the topical peri-arterial application of porcine elastase solution with $20 \mathrm{~min}$ induction. This method is easy, efficient and reproducible with aneurysm stability over 21 days. It is also helpful for the research about endovascular techniques and devices.

\section{Abbreviations}

CD31: Platelet endothelial cell adhesion molecule-1, PECAM-1/CD31; DSA: Digital subtraction angiography; EVG: Elastic van-Gieson; H\&E: Hematoxylin and eosin stain; IVDSA: Intravenous digital subtraction angiography; PBS: Phosphor buffered saline; RCCA: Right common carotid artery; SMCs: Smooth muscle cells; a-SMA: Alpha smooth muscle Actin

\section{Acknowledgements}

Not applicable.

\section{Funding}

No funding.

\section{Availability of data and materials}

The datasets generated during and analysed during the current study are available from the corresponding author on reasonable request.

\section{Authors' contributions}

Conception and design: X-GF, G-QJ,Z-FL. Acquisition of data: G-QJ, Z-FL. Analysis and interpretation of data: X-GF, G-QJ, Z-BL. Drafting the article: G-QJ, X-CJ. Critically revising the article: X-GF, G-QJ. Reviewed the submitted version of the manuscript: X-GF, S-SX. Performed the statistical analysis: G-QJ, Z-FL. Study supervision: X-GF. All authors read and approved the final manuscript. 


\section{Ethics approval and consent to participate}

This study was approved by the Institutional Review Board of Wannan medical College (Research Ethics Committee of Wannan medical College, Permit Number: 2015,05).

\section{Consent for publication}

Not applicable.

\section{Competing interests}

The authors declare that they have no competing interests.

\section{Author details}

'Department of Neurosurgery, Second Affiliated Hospital of Wannan Medical College, Wuhu, China. ${ }^{2}$ Department of Neurosurgery, Changhai Hospital, Second Military Medical University, Shanghai, China. ${ }^{3}$ Department of Neurosurgery, First Affiliated Hospital of Wannan Medical College, Wuhu 241000, China

Received: 19 October 2016 Accepted: 8 August 2017

Published online: 05 October 2017

\section{References}

1. Park SH, Yim MB, Lee CY, Kim E, Son El. Intracranial Fusiform aneurysms: It's pathogenesis, clinical characteristics and managements. J Korean Neurosurg Soc. 2008;44:116-23.

2. Day AL, Gaposchkin CG, Yu CJ, Rivet DJ, Dacey RG. Spontaneous fusiform middle cerebral artery aneurysms: characteristics and a proposed mechanism of formation. J Neurosurg. 2003:99:228-40.

3. Selviaridis P, Spiliotopoulos A, Antoniadis C, Kontopoulos V, Foroglou G. Fusiform aneurysm of the posterior cerebral artery: report of two cases. Acta Neurochir. 2002;144:295-9.

4. Al-Yamany M, Ross IB. Giant fusiform aneurysm of the middle cerebral artery: successful Hunterian ligation without distal bypass. Br J Neurosurg. 1998;12:572-5.

5. Nonn A, Kirschner S, Figueiredo G, Kramer M, Nikoubashman O, et al. Feasibility, safety, and efficacy of flow-diverting stent-assisted microsphere Embolization of Fusiform and sidewall aneurysms. Neurosurgery. 2015;77: 126-35.

6. Shi WY, Gu JP, Li MH, Yan L, He X. The predictors of endoleaks after endovascular repair of experimentally produced fusiform carotid aneurysm in canine. Minim Invasive Ther Allied Technol. 2016;25:99-106.

7. Yan L, Shi WY, Wang D, Zhu YQ, Tan HQ, et al. Application of the Willis covered stent using the telescopic technique for the treatment of fusiform aneurysm in a canine model. Neurol India. 2013;61:45-50.

8. Isenburg JC, Simionescu DT, Starcher BC, Vyavahare NR. Elastin stabilization for treatment of abdominal aortic aneurysms. Circulation. 2007:115:1729-37.

9. Yalcin M, Yiginer O, Eroglu M, Sehirli O, Berber U, et al. Effect of hyperbaric oxygen treatment in a rat model of abdominal aortic aneurysm. Undersea Hyperb Med. 2014;41:9-15.

10. Gacchina C, Brothers T, Ramamurthi A. Evaluating smooth muscle cells from $\mathrm{CaCl} 2$-induced rat aortal expansions as a surrogate culture mode for study of elastogenic induction of human aneurysmal cells. Tissue Eng A. 2011;17:1945-58.

11. Bi Y, Zhong H, Xu K, Zhang Z, Qi X, et al. Development of a novel rabbit model of abdominal aortic aneurysm via a combination of periaortic calcium chloride and elastase incubation. PLoS One. 2013;8:e68476.

12. Fahed R, Raymond J, Ducroux C, Gentric JC, Salazkin I,et al. Testing flow diversion in animal models: a systematic review. Neuroradiology. 2016;58: 375-82.

13. Anidjar S, Salzmann $J$, Gentric D, Lagneau P, Camilleri JP, et al. Elastase-induced experimental aneurysms in rats. Circulation. 1990;82: 973-81.

14. Altes TA, Cloft HJ, Short JG, DeGast A, Do HM, et al. 1999 ARRS executive council award. Creation of saccular aneurysms in the rabbit: a model suitable for testing endovascular devices. American Roentgen Ray Society. AJR Am J Roentgenol. 2000;174:349-54.

15. Nuki Y, Tsou TL, Kurihara C, Kanematsu M, Kanematsu Y, et al. Elastaseinduced intracranial aneurysms in hypertensive mice. Hypertension. 2009;54: $1337-44$.
16. Cloft HJ, Altes TA, Marx WF, Raible RJ, Hudson SB, et al. Endovascular creation of an in vivo bifurcation aneurysm model in rabbits. Radiology. 1999;213:223-8.

17. Ding YH, Kadirvel R, Dai D, Kallmes DF. Creation of bifurcation-type elastaseinduced aneurysms in rabbits. Am J Neuroradiol. 2013;34:E19-21.

18. Reinald N, Fournier B, Naveau A, Couty L, Lemitre M, et al. Fusiform aneurysm model in rabbit carotid artery. J Vasc Res. 2010;47:61-8.

19. Hoh BL, Rabinov JD, Pryor JC, Ogilvy CS. A modified technique for using elastase to create saccular aneurysms in animals that histologically and hemodynamically resemble aneurysms in human. Acta Neurochir. 2004;146: 705-11.

20. Hosaka K, Downes DP, Nowicki KW, Hoh BL. Modified murine intracranial aneurysm model: aneurysm formation and rupture by elastase and hypertension. J Neurointervent Surg. 2014;6:474-9.

21. Hoh BL, Velat GJ, Wilmer EN, Hosaka K, Fisher RC, et al. A novel murine elastase saccular aneurysm model for studying bone marrow progenitorderived cell-mediated processes in aneurysm formation. Neurosurgery. 2010; 66:544-50.

\section{Submit your next manuscript to BioMed Central and we will help you at every step:}

- We accept pre-submission inquiries

- Our selector tool helps you to find the most relevant journal

- We provide round the clock customer support

- Convenient online submission

- Thorough peer review

- Inclusion in PubMed and all major indexing services

- Maximum visibility for your research

Submit your manuscript at www.biomedcentral.com/submit
) Biomed Central 